\title{
Supporting Information for \\ Investigating Primary Charge Separation in the Reaction Center of Heliobacterium modesticaldum
}

\author{
Journal of Physical Chemistry $B$
}

Moritz Brütting, Johannes M. Foerster, and Stephan Kümmel

Theoretical Physics IV • University of Bayreuth

\section{Contents}

$\begin{array}{ll}\text { Computational Methods } & \text { S1 }\end{array}$

Structure Preparation . . . . . . . . . . . . . . . . S1

Born-Oppenheimer Molecular Dynamics . . . . . . . . . . . . . . . S2

$\begin{array}{ll}\text { Results and Discussion } & \text { S2 }\end{array}$

Coupling of the Bacteriochlorophyll-Pigments . . . . . . . . . . . S S2

Excited States of the SPP-Acc System . . . . . . . . . . . . . . . S7

Numerical Tests . . . . . . . . . . . . . . . . . . . S7

Protein Environment . . . . . . . . . . . . . . . . . . S9

Born-Oppenheimer Molecular Dynamics . . . . . . . . . . . . . . . . S10

Experimental Spectra . . . . . . . . . . . . . . . . . . . S13

$\begin{array}{ll}\text { Tables of the Excitation Spectra } & \text { S13 }\end{array}$

$\begin{array}{ll}\text { References } & \text { S13 }\end{array}$

\section{Computational Methods}

\section{Structure Preparation}

As a complement to the main text, we describe the preparation of the structure of the SPP-Acc system in the following. To reduce the computational effort the phythyl tails of the Bcl pigments, which just serve to keep the pigments in position, are not included in our calculations. Therefore, these hydrocarbon chains are cropped from their ester linkages to the side chains of the bacteriochlorin rings and replaced by an $\mathrm{H}$ atom. Furthermore, the bonds of the amino acid residues to the polypeptide chains are cut between $\mathrm{C}_{\alpha}$ and $\mathrm{C}_{\beta}$ and replaced by further $\mathrm{H}$ atoms. This procedure keeps the terminal $\mathrm{C}_{\beta}$ saturated. The positions of the $\mathrm{H}$ atoms, which cannot be resolved by the X-ray analysis, are energetically optimized using the CHARMM force field as described in [1]. Thereby, the positions of the other atoms $(\mathrm{C}, \mathrm{N}, \mathrm{O}, \mathrm{Mg})$ are kept fixed. 


\section{Born-Oppenheimer Molecular Dynamics}

For the BOMD simulations we use the Ahlrichs def2-SV(P) Gaussian basis set, the $\mathrm{m} 3$ grid, and the resolution-of-the-identity approximation. The size of the BOMD time steps is $0.12 \mathrm{fs}$, which is small enough to resolve the fast vibrations of the $\mathrm{H}$ atoms. A Nosé-Hoover thermostat is used to keep the pseudo-temperature around $300 \mathrm{~K}$. The thermostat relaxation time is $0.73 \mathrm{fs}$.

As fixing atomic coordinates is not supported by the standard BOMD implementation of TURBOMOLE, we use a modified version of a TURBOMOLE in-house tool originally developed by Arnim Hellweg (TURBOMOLE GmbH, Karlsruhe) to perform the MD cycles. In order to make our calculations transparent, we describe its functionality in the following. In each BOMD time step three separate computational tasks are performed by different (standard) TURBOMOLE routines: determining the electronic ground state corresponding to the current positions of the nuclei from a DFT self consistent iteration (ridft), calculating the (classical) forces on the nuclei resulting from this particular electronic configuration (rdgrad), and, finally, updating the positions and velocities of the nuclei (frog). The routines exchange the information on positions and velocities of the nuclei via specific files. Hence, by zeroing the velocities and forces corresponding to the fixed atoms in these files between the second and third computational step, the mechanical constraints can be ensured in a straightforward way. This particular functionality is not integrated into the standard BOMD implementation of TURBOMOLE.

\section{Results and Discussion}

\section{Coupling of the Bacteriochlorophyll-Pigments}

As a complement to the discussion of the excitation spectrum of the SPP-Acc system in the main text we analyze the spectra of its constituent subsystems, SPP-His and Acc-Gln, in this section. SPP-His consists of two Bcl $g$ ' pigments which are each coordinated by His 537 ("Bcl g'-His"). Similarly, Acc-Gln is composed of two Bcl $g$ pigments which are each coordinated by a water molecule H-bonded by Gln 458 ("Bcl $g$-Gln"). In Figure S1 (a) the spectra of SPP-His and Bcl $g$ '-His, and in (b) the spectra of Acc-Gln and Bcl $g-\mathrm{Gln}$ are compared.

We begin the discussion with case (b) as it is somewhat more transparent. Strikingly, the $\mathrm{Q}_{\mathrm{y}}$ and $\mathrm{Q}_{\mathrm{x}}$ transitions in the spectrum of the individual $\mathrm{Bcl} g-\mathrm{Gln}$ correspond to two excitations with virtually the same energies in the spectrum of the Acc-Gln aggregate. To rationalize this result it is instructive to think of the coupled excitations of Acc-Gln as resulting from combined excitations of the individual $\mathrm{Bcl} g-\mathrm{Gln}$ subsystems. This approximation is appropriate if the subsystems can be treated as well separated entities. For estimating whether this is the case one can compare the intermolecular distance of the Accs pigments (19.5 $\AA$ center-to-center) to the "diameter" of one bacteriochlorin ring ( $\sim 7 \AA$ ). The character of the coupling among the subunits is revealed by analyzing the transition densities for the excitations of Acc-Gln. They are depicted in Figure S2. For the 


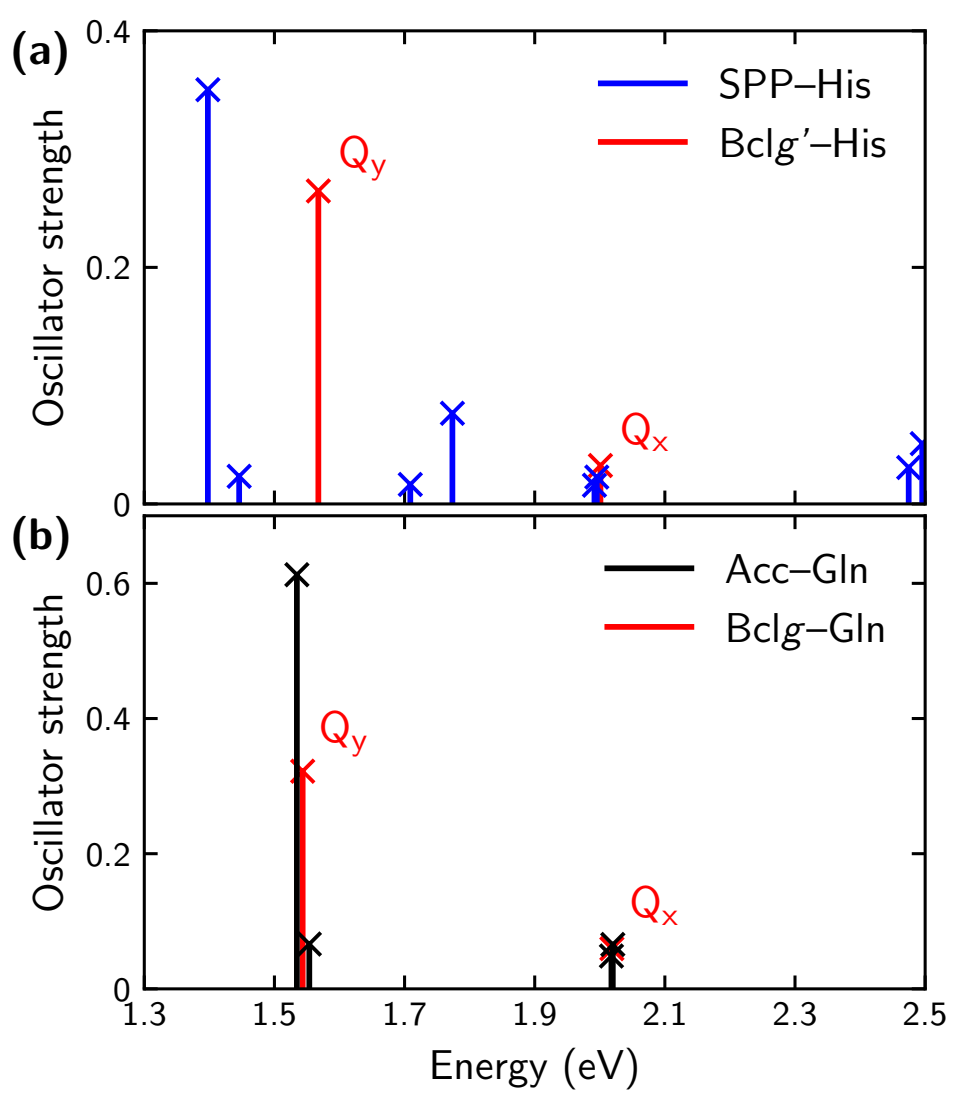

Figure S1: (a) Excitation spectra of SPP-His and a single Bcl $g^{\prime}-\mathrm{His}$, calculated with the 6-31G(d,p) basis set. (b) Excitation spectra for Acc-Gln and a single Bcl g-Gln. The $Q_{y}$ and $\mathrm{Q}_{\mathrm{x}}$ transitions of $\mathrm{Bcl} g^{\prime}-\mathrm{His}$ and $\mathrm{Bcl} g-\mathrm{Gln}$, respectively, are indicated.

$\mathrm{Q}_{\mathrm{x}}$ excitations ((c) and (d)) the transition density in each case is predominantly localized on one of the $\mathrm{Bcl} g$-Gln and the other chromophore carries much less transition density. In these cases, exciting Acc-Gln in fact corresponds to exciting predominantly just one of its Bcl $g$-Gln subunits. By contrast, the $\mathrm{Q}_{\mathrm{y}}$ transition densities (cf. Figure S2 (a) and (b)) are fully delocalized over both rings. It turns out that the transition dipoles that one can associate with the individual Bcl $g$-Gln subunits are ordered roughly parallel for the transition with lower energy and higher oscillator strength, and antiparallel for the transition with higher energy and lower oscillator strength. This corresponds to the well-known concept of a J-aggregate [2,3]: The delocalized excited state with lower (higher) energy results from the symmetric (antisymmetric) coupling of the excitations of the individual subunits.

Similar coupling mechanisms are also observed for the spectrum of SPP-His, cf. Figure S1 (a). However, here the situation is more complex due to the significantly lower inter-pigment distance - the SPP pigments have a center-to-center distance of only $5.9 \AA$ 

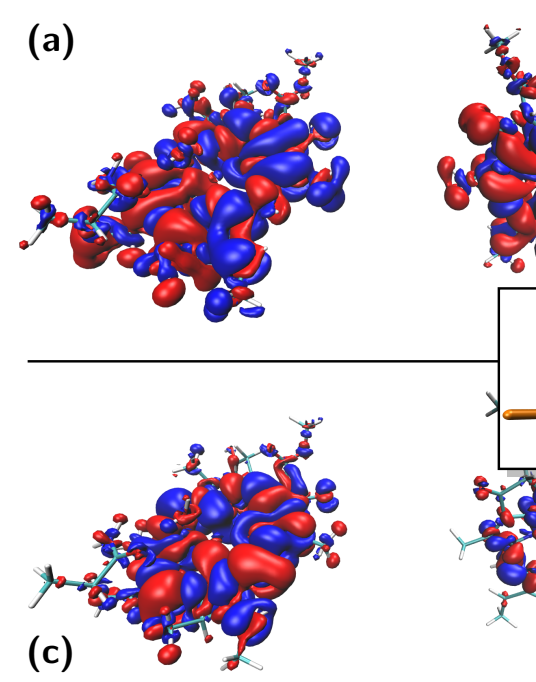

(b)
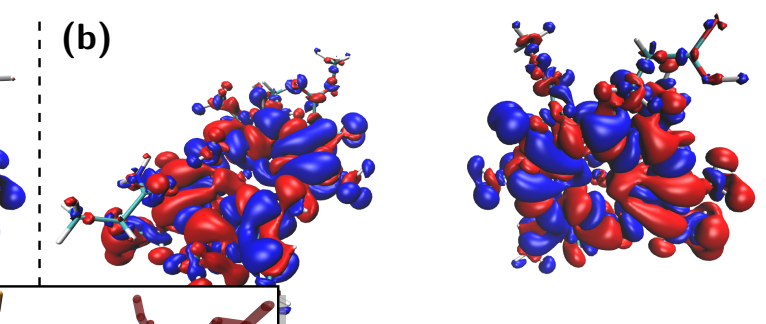

Figure S2: Transition densities of the coupled $Q_{y}\left((\mathbf{a})\right.$ and (b)) and $Q_{x}(\mathbf{( c )}$ and (d)) transitions of Acc-Gln (in order of increasing excitation energy). Isosurfaces with isovalues of $\pm 0.0002 \mathrm{a}_{0}^{-3}$ are displayed. The isosurface corresponding to the positive (negative) isovalue is colored red (blue). The inset schematically shows the molecules without transition densities to clarify the chosen point of view.
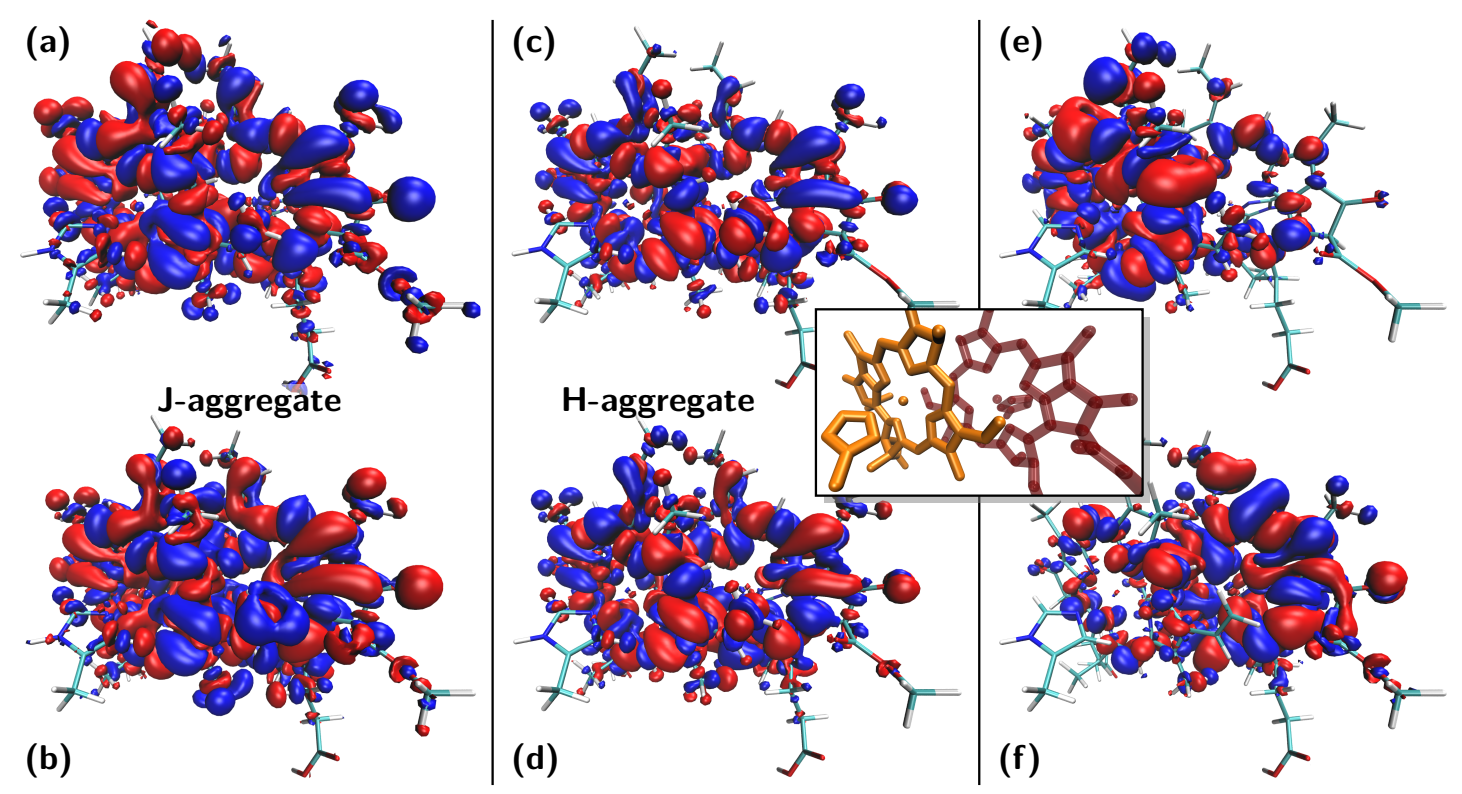

Figure S3: Transition densities of the coupled $\left.Q_{y}(\mathbf{a})-(\mathbf{d})\right)$ and $Q_{x}((\mathbf{e})$ and $(\mathbf{f}))$ transitions of SPP-His (in order of increasing excitation energy). The isosurface representation is the same as in Figure S2. The inset schematically shows the molecules without transition densities to clarify the chosen point of view. 
- and, hence, stronger coupling of the individual Bcl $g$ '-His subunits. Upon coupling, the $\mathrm{Q}_{\mathrm{y}}$ excitation splits into four coupled excitations, which group into two pairs consisting of one excitation with higher and one with lower oscillator strength each. The excitation energies of these pairs are red-shifted (blue-shifted) by $0.15 \mathrm{eV}(0.17 \mathrm{eV})$ compared to the $\mathrm{Q}_{\mathrm{y}}$ transition of the isolated $\mathrm{Bcl} g$ '-His subunit. The $\mathrm{Q}_{\mathrm{x}}$ transition splits, as before, into two coupled excitations of similar energy. The corresponding transition densities are depicted in Figure S3. Once again, the $\mathrm{Q}_{\mathrm{x}}$ transition densities of the aggregate are predominantly localized on single Bcl $g$ '-His entities, i. e., are rather uncoupled. The coupling of the $\mathrm{Q}_{\mathrm{y}}$ excitations can be rationalized by the theory of molecular aggregates again. Here, the coupling of the $\mathrm{Q}_{\mathrm{y}}$ pair with lower energy is approximately of J-type, whereas that of the pair with higher energy is of H-type. In the latter case the delocalized excited state with lower (higher) energy results from antisymmetric (symmetric) coupling. We attribute the fact that both J-type and H-type couplings emerge to the strong coupling of the SPP.
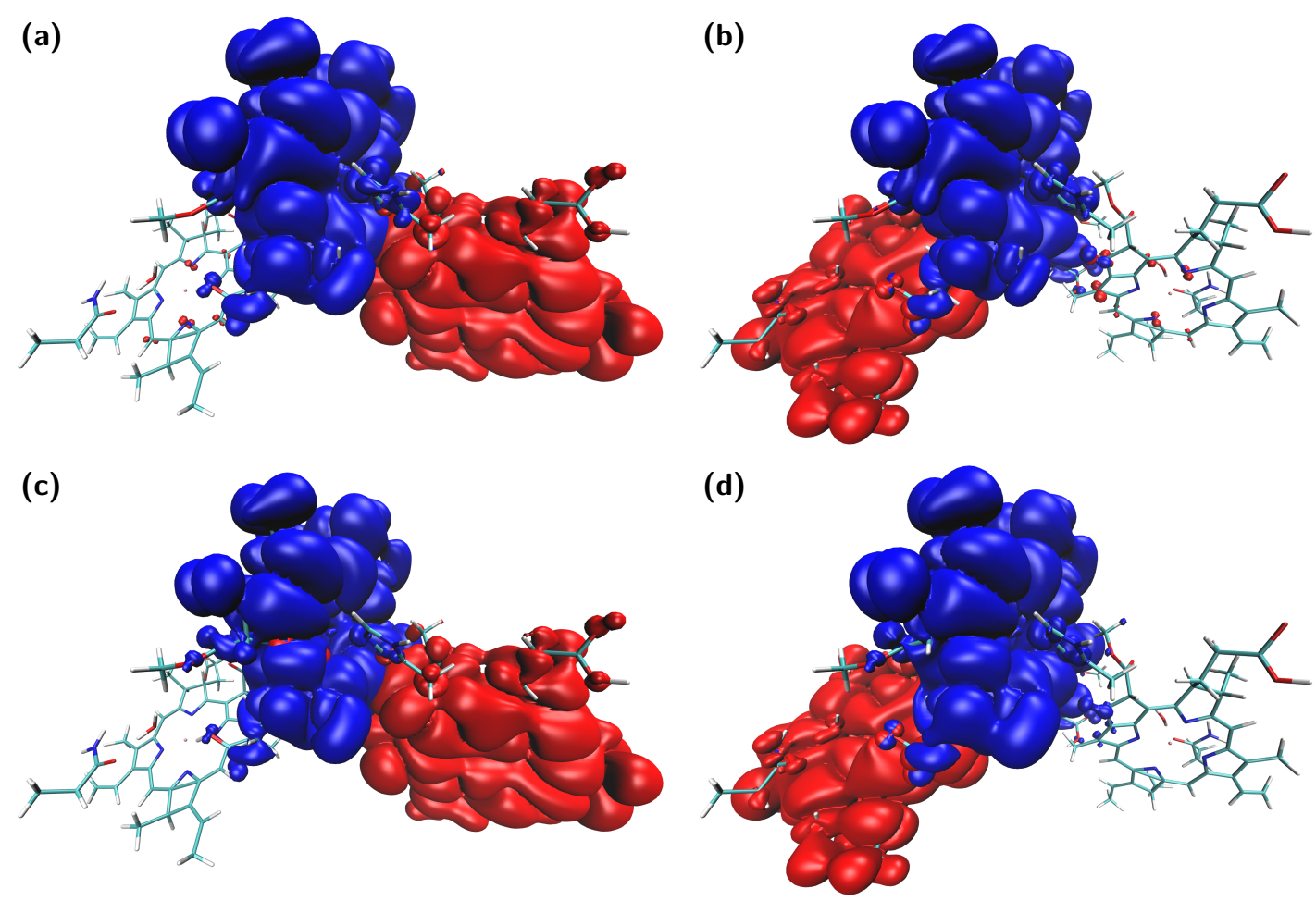

Figure S4: Hole (blue) and electron (red) densities of the first NTO pairs of the four forward-CT states identified in the spectrum of SPP-Acc. The arrangement of the subfigures is in the order of increasing excitation energy: (a) $1.882 \mathrm{eV}$, (b) $1.894 \mathrm{eV}$, (c) $2.240 \mathrm{eV}$, and (d) $2.260 \mathrm{eV}$. In all cases the isosurfaces represent an isovalue of $4 \cdot 10^{-6} \mathrm{a}_{0}^{-3}$. 


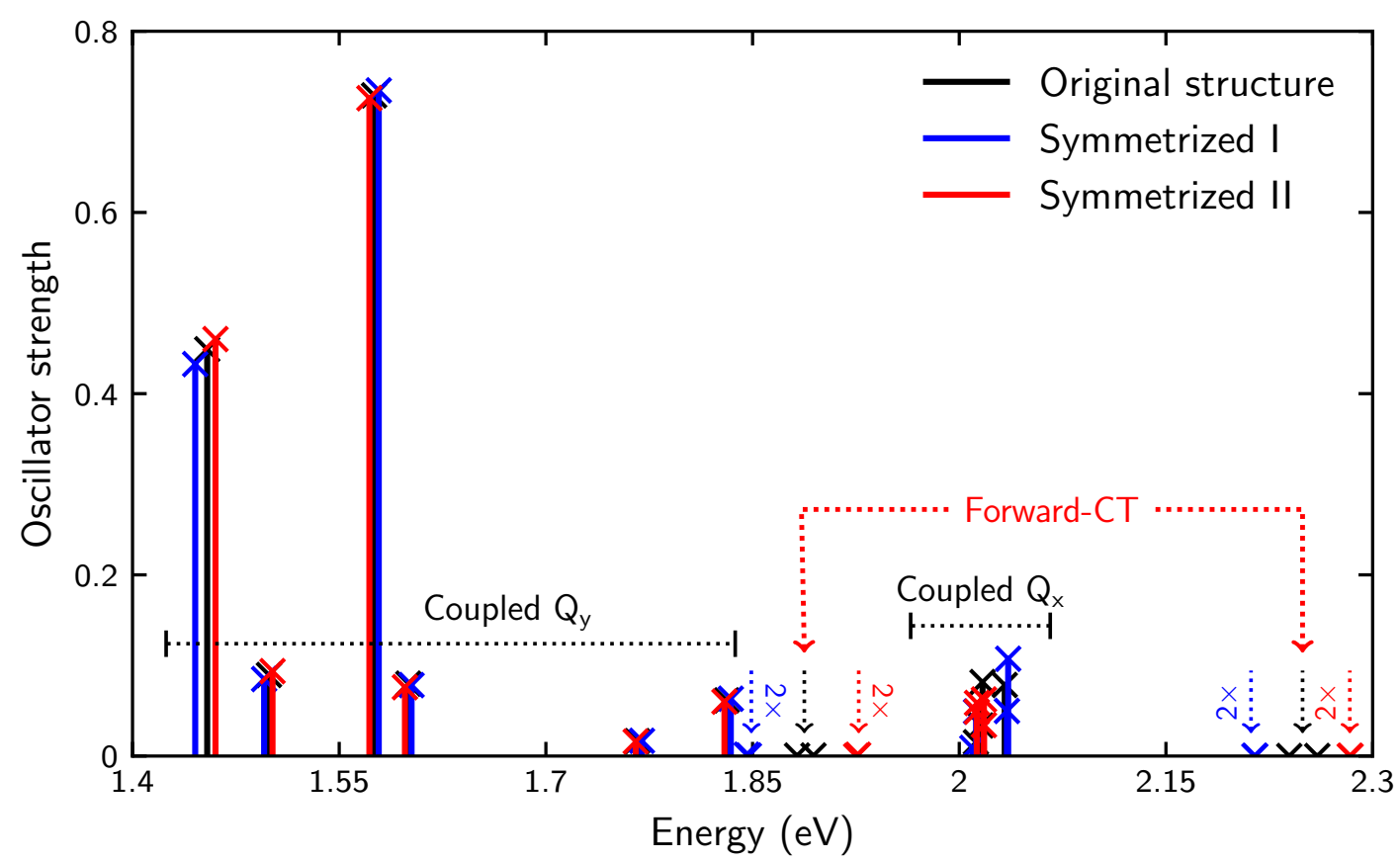

Figure S5: Comparison of the excitation spectra emerging from the original structure of SPPAcc and its two symmetrized variants ("Symmetrized I" and "Symmetrized II"), calculated with the 6-31G basis set. The character of the states is indicated as described in the main text. Note that for the symmetrized structures the forward-CT states emerge in pairs of virtually identical excitation energies (as indicated).

(a)

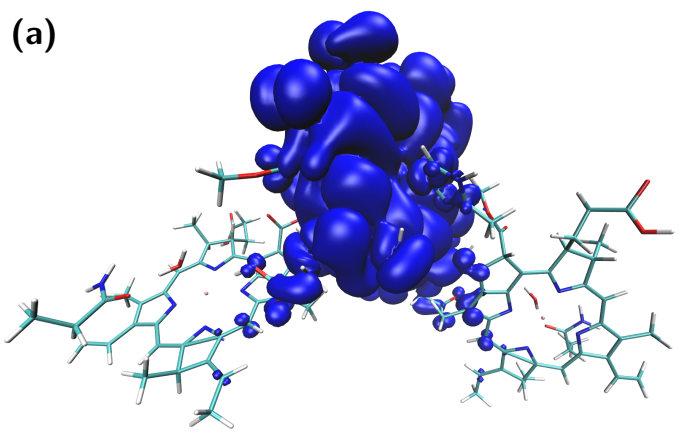

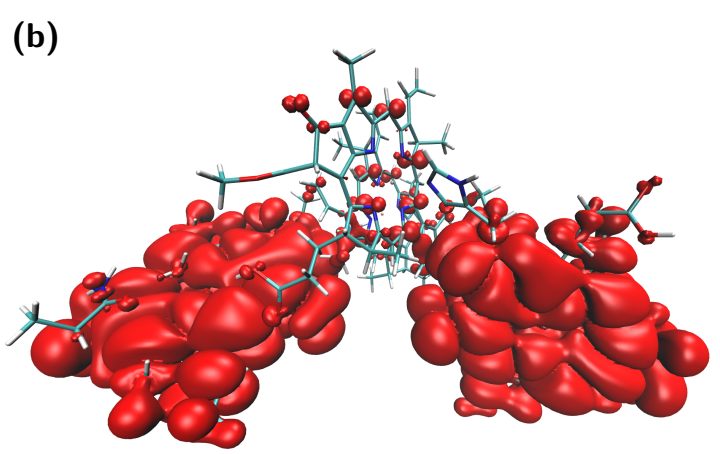

Figure S6: (a) Hole and (b) electron density of the first NTO pair of the lowest forward-CT state (at $1.846 \mathrm{eV}$ ) in the spectrum of one symmetrized structure of SPP-Acc ("Symmetrized I"). The corresponding NTOs of the other forward-CT states (of both symmetrized structures) look virtually identical. The isosurface representation is the same as in Figure S4. 


\section{Excited States of the SPP-Acc System}

The forward-CT excitations in the SPP-Acc system emerge in pairs of two states, cf. Figure 4 (main text). Figure S4 provides additional information on the NTOs of these forward-CT states. This illustrates that $\mathrm{CT}$ is directed along different branches of the HbRC for the two states of such a pair. Otherwise the shape of the NTOs barely changes.

We argue in the main text, that the small energy gap of $\sim 0.02 \mathrm{eV}$ between the individual excitations of each forward-CT state pair arises from small structural asymmetries between the different branches of the HbRC. We are able to verify this assumption by explicitly symmetrizing the structure of SPP-Acc. We generate the symmetrized structures by splitting the original unsymmetric structure into two halves, each consisting of one half of the SPP-His and Acc-Gln. We then rotate each of these two parts by $180^{\circ}$ around the $\mathrm{C} 2$ symmetry axis to generate two symmetric versions of the SPP-Acc system ("symmetrized I" and "symmetrized II"). These symmetrized versions differ from the crystal structure and among each other merely in the position of the $\mathrm{H}$ atoms. The corresponding excitation spectra ("Symmetrized I" and "Symmetrized II") are compared in Figure S5 to the one emerging from the original structure that exhibits the slight asymmetry. The coupled $\mathrm{Q}_{\mathrm{y}}$ and $\mathrm{Q}_{\mathrm{x}}$ excitations are barely influenced by the symmetrization process. In contrast, the forward-CT states exhibit qualitative differences. The small energetic difference within each pair of excitations disappears, and the forward-CT excitation energies for the symmetrized structures are red-shifted (Symmetrized I) or blue-shifted (Symmetrized II), respectively, by $\sim 0.04 \mathrm{eV}$ on average, compared to the original spectrum. An NTO analysis reveals that also the character of the forward-CT excitations changes. An example is depicted in Figure S6; the corresponding NTOs of all other forward-CT states look virtually identical. The electron density is delocalized equally over both Acc pigments for the symmetrized structures.

\section{Numerical Tests}

Q-CHEM represents functions by Gaussian basis sets and we used the 6-31G, 6-31G(d,p), and $6-311 G(d, p)$ Pople basis sets in our work. In Figure S7 we demonstrate the influence that the basis set has on the excitations of the SPP-Acc system. It turns out that the variation of the basis set leaves the order of the excitations and their relative spectral separation unchanged and merely induces a global red-shift of the excitation energies. Its magnitude decreases with increasing basis set size, from $0.05 \mathrm{eV}$ between $6-31 \mathrm{G}$ and 6-31G(d,p) to $0.02 \mathrm{eV}$ between $6-31 \mathrm{G}(\mathrm{d}, \mathrm{p})$ and $6-311 \mathrm{G}(\mathrm{d}, \mathrm{p})$. Therefore, we conclude that already the $6-31 \mathrm{G}$ basis set is sufficient to identify and observe the relative spectral positions of the forward-CT states within the spectrum.

We note that in order to ensure proper convergence of the self-consistent iterations that provide the orbitals and eigenvalues that are used in the Casida equations, the corresponding convergence threshold needs to be set to cis_convergence 7 .

As another numerical test, we explored the influence of adding or removing the coordinating His or $\mathrm{H}_{2} \mathrm{O}-\mathrm{Gln}$, respectively, in the calculations, cf. Figure S8. We find 


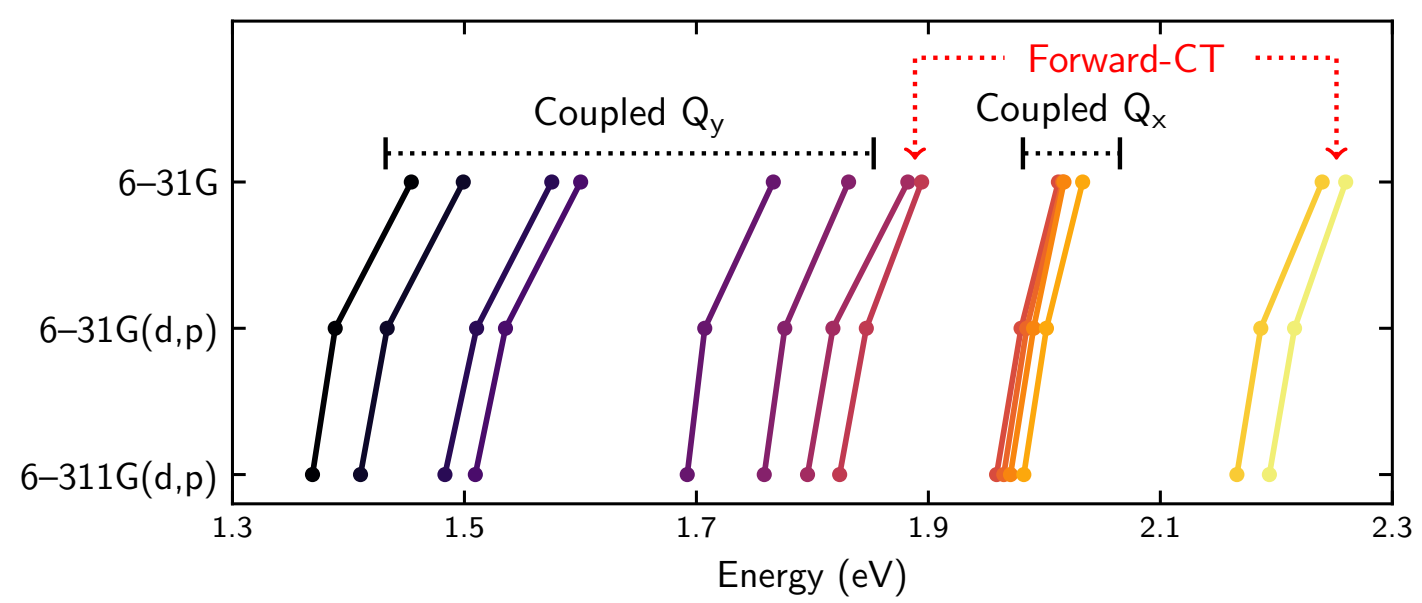

Figure S7: Excitation energies of the SPP-Acc system calculated with different basis sets: 6-31G, 6-31G $(d, p)$, and 6-311G $(d, p)$. The character of the excitations is indicated as in the main text. The lines connect corresponding excitations. Upon change of the basis set, the average shift of the excitation energies is -0.05 (top) and $-0.02 \mathrm{eV}$ (bottom).

that the coupled $\mathrm{Q}_{\mathrm{y}}$ and $\mathrm{Q}_{\mathrm{x}}$ excitations are barely influenced by these amino acid residues, as they are shifted by only $\lesssim 0.02$ and $0.06 \mathrm{eV}$, respectively. The forward-CT states exhibit more significant energy variations. Upon addition of His they are red-shifted by $0.50 \mathrm{eV}$. The effect of $\mathrm{H}_{2} \mathrm{O}-\mathrm{Gln}$ is smaller, yet still significant with a blue-shift by $0.16 \mathrm{eV}$. Hence, the influence of His and $\mathrm{H}_{2} \mathrm{O}-\mathrm{Gln}$ is important regarding the position of the CT states in the spectrum.

For all calculations presented in this work the range separation parameter $\omega$ of $\omega \mathrm{PBE}$ has been set to $\omega_{a}=0.171 \mathrm{a}_{0}^{-1}$. This value stems from applying the optimal tuning procedure to a single $\mathrm{Bcl} a$ [1]. The central building block of our particular system is $\mathrm{Bcl} g$ ', though. Given the similarity between these molecules it is reasonable to assume that optimal tuning for $\mathrm{Bcl} g$ ' leads to a comparable range-separation parameter. We have verified this and find $\omega_{g}=0.160 \mathrm{a}_{0}^{-1}$ when we optimally tune for $\mathrm{Bcl} g^{\prime}$. We also compared the spectrum of SPP-Acc calculated with $\omega_{a}$ to the one calculated with $\omega_{g}$ to clarify the influence that the range-separation parameter has on the excitation energies. This comparison is shown in Figure S9. It turns out that decreasing the range separation parameter induces a small red-shift, up to $0.07 \mathrm{eV}$ for the forward-CT states. Otherwise, the spectra hardly change. We therefore conclude that the relevant physical effects that we focus on in our study are described correctly with either choice of range-separation parameter.

We would like to stress that we deliberately do not tune the range-separation parameter anew for each (sub-)system, but, e. g., calculate SPP-Acc, SPP-His, and Acc-Gln with the same value of $\omega$. If the parameter would be tuned anew for each system, the calculations would effectively use different exchange-correlation approximations and could not be compared on the same footing. Furthermore, previous studies $[4,5]$ showed that 


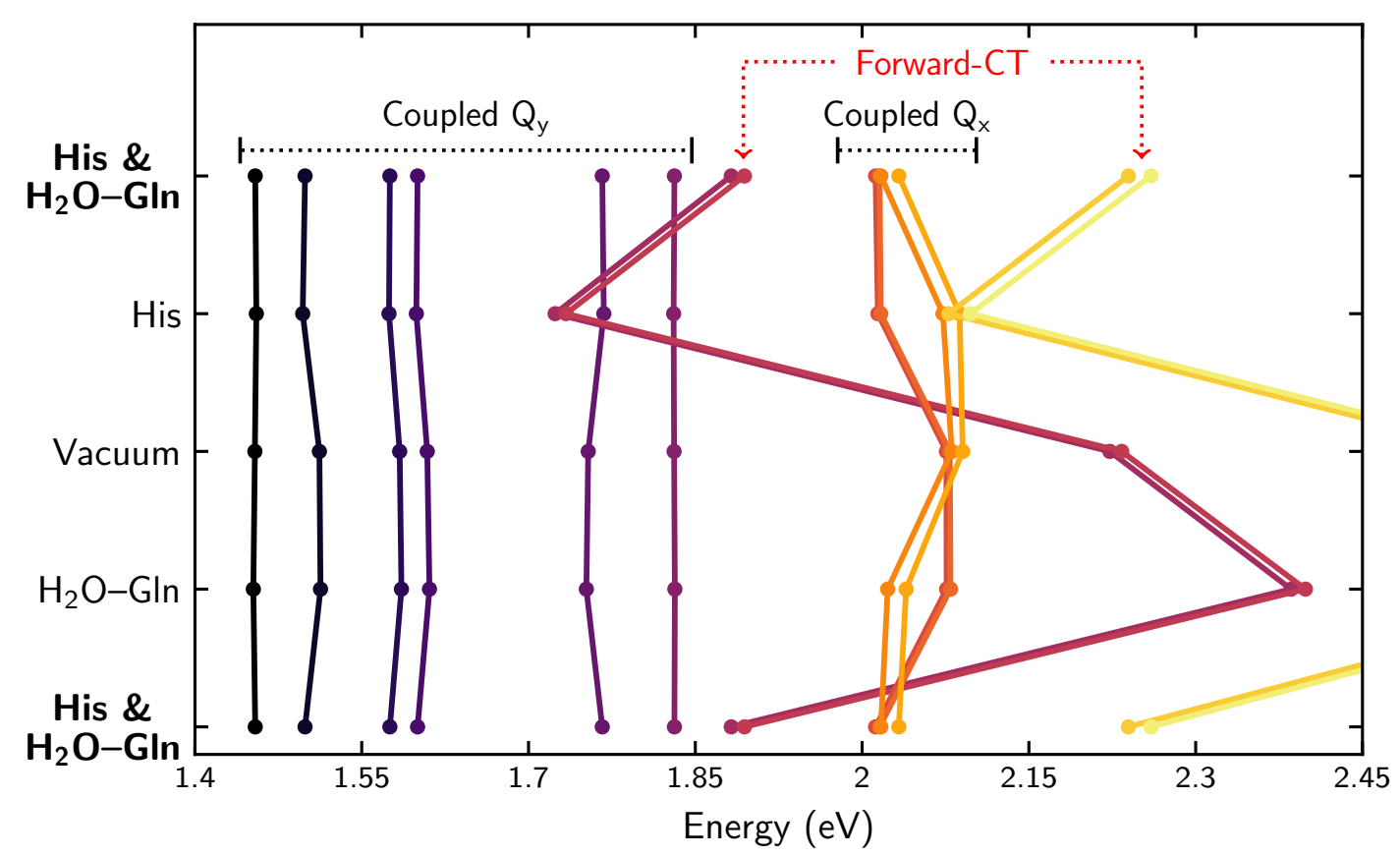

Figure S8: Influence of the coordinating molecules on the excitation energies of the SPP-Acc system, calculated with the 6-31G basis set. The configurations considered are: without any coordinating molecules ("vacuum"), with $\mathrm{His}$, with $\mathrm{H}_{2} \mathrm{O}-\mathrm{Gln}$, and with both $\mathrm{His}$ and $\mathrm{H}_{2} \mathrm{O}-\mathrm{G}$ ln (bold label, shown twice for ease of comparison). The latter corresponds to the spectrum shown in the main text. The order of the excitations changes, as indicated by the connecting lines.

tuning for conjugated systems of increasing size can reveal artifacts due to the semi-local functional pieces in the range-separated hybrid, leading to unrealistically low values of the range-separation parameter. Effectively, the range-separated hybrid then turns into an almost semi-local functional, with the corresponding shortcomings, e.g., regarding the description of CT excitations.

\section{Protein Environment}

In the main text, we discuss the influence of the protein environment by comparing the excitation spectrum of the SPP-Acc system embedded into a particular selection of amino acid residues, defined in Table 1 (main text), to that of the bare SPP-Acc system. We further mention that certain polar or aromatic amino acids from this selection, i. e., Trp, Thr, and Tyr, have the largest influence on the excitation spectrum. Figure S10 provides additional information on this point by comparing the excitation energies of the SPP-Acc system within three different amino acid selections - Trp; Trp, Thr, and Tyr; the full amino acid selection - to those of the SPP-Acc system alone. This graphical 


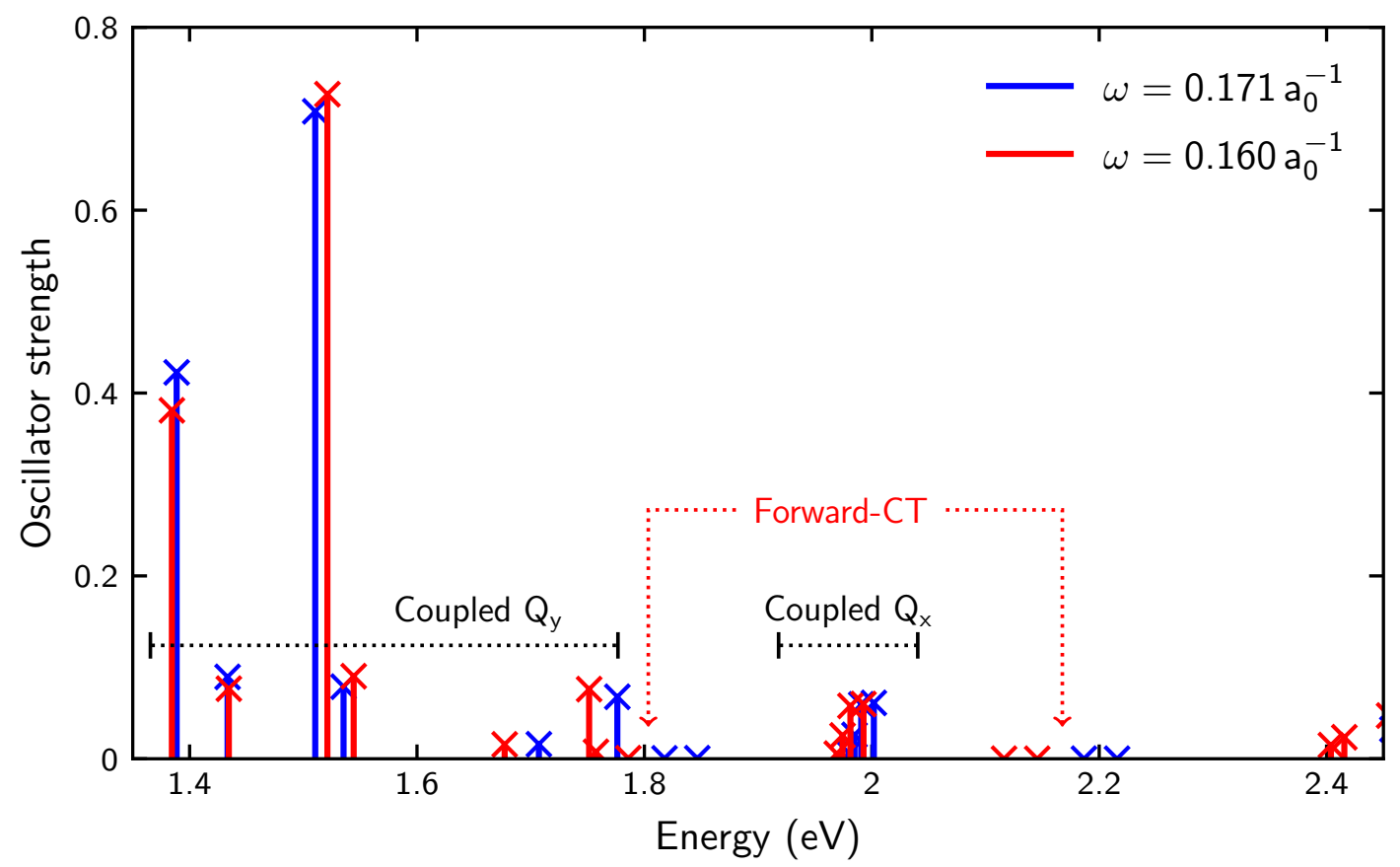

Figure S9: Excitation spectrum of SPP-Acc for different range separation parameters: $\omega=0.171 \mathrm{a}_{0}^{-1}$ (from [1], used throughout this work) and $\omega=0.160 \mathrm{a}_{0}^{-1}$ (from OT of a single $\left.\mathrm{Bcl} g^{\prime}\right)$, both calculated with the $6-31 \mathrm{G}(\mathrm{d}, \mathrm{p})$ basis set. The character of the states is indicated as in the main text.

representation supports our conclusions from the main text, i. e., adding Trp alone redshifts the forward-CT states by $\sim 0.14 \mathrm{eV}$. Adding both Thr and Tyr induces a blue-shift of similar size.

We also checked which influence the phythyl tails of the Bcl $g$ ' from the SPP, which coordinate the Accs, have on the excitation spectrum, cf. Figure S10. We find that they have little impact on the excitation spectrum as they only induce an overall shift $\lesssim 0.04 \mathrm{eV}$.

\section{Born-Oppenheimer Molecular Dynamics}

In the main text, we discuss the influence of the nuclear motion on the excitation spectrum of SPP-Acc by comparing a histogram of the forward-CT states to an ensemble-averaged excitation spectrum. The latter constitutes a transparent way of representing the huge number of excitation spectra for the different geometries from the BOMD simulation in an efficient manner. However, it does not provide insights into the nature of the underlying excitations, i. e., whether their character is similar to $\mathrm{Q}_{\mathrm{y}}$ or $\mathrm{Q}_{\mathrm{x}}$ transitions, and their time dynamics. A pragmatic way to shed light on this matter is to plot the time evolution of certain energy levels which represent the main features of the BOMD-based 


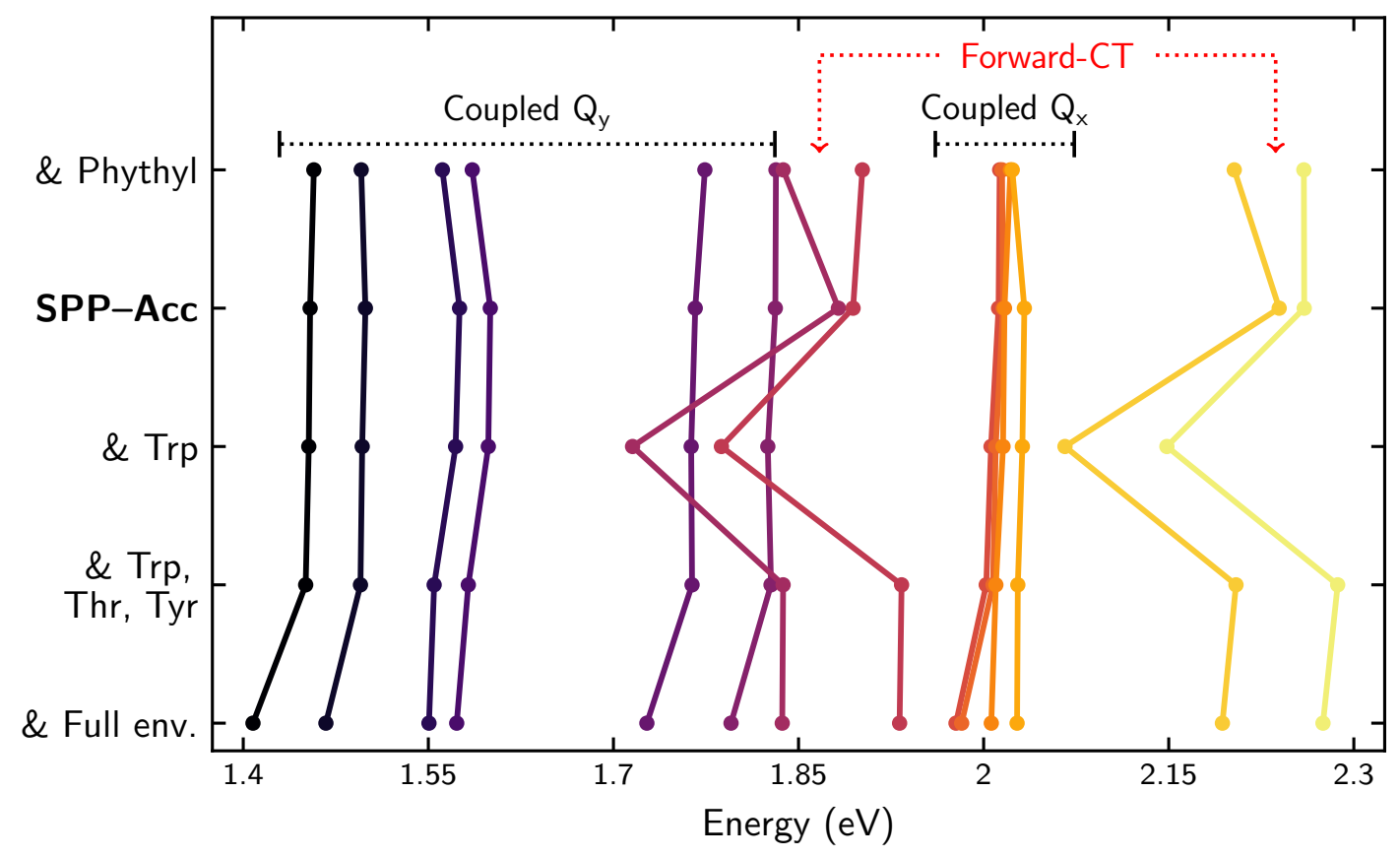

Figure S10: Influence of the environment on the excitation energies of SPP-Acc, calculated with the 6-31G basis set. Displayed are the energies of SPP-Acc alone (bold label) as well as combined with the phythyl tails of the SPP Bcl $g$ 's (above) and certain amino acid selections: Trp; Trp, Thr, and Tyr; the full environment amino acid selection (cf. text). The order of the excitations changes, as indicated by the connecting lines.

excitation spectra. To that end we define three energy levels - named main $Q_{y}$, mean $Q_{x}$, and lowest forward-CT - whose time evolution is depicted in Figure S11.

The main $Q_{y}$ energy level is defined as the energy of the excitation with the highest oscillator strength. This will be one of the coupled $\mathrm{Q}_{\mathrm{y}}$ excitations. We choose this excitation for representing the typical $\mathrm{Q}_{\mathrm{y}}$ energy, because in our experience the coupled $\mathrm{Q}_{\mathrm{y}}$ excitations are typically dominated by a single excitation. In the case of the coupled $\mathrm{Q}_{\mathrm{x}}$ excitations the picture is different. Normally, there is no single dominant excitation. In our experience, however, the average of the two $\mathrm{Q}_{\mathrm{x}}$ excitations with the highest oscillator strengths represents this type of excitation well. We refer to this average energy as the mean $Q_{x}$ energy level. On the technical side, we determine these two excitation energies by first excluding the six excitations with lowest energy (which will be the coupled $\mathrm{Q}_{\mathrm{y}}$ 's), and then searching among the remaining excitations for the two ones with the oscillator strengths. Obtaining the lowest forward-CT energy is straightforward as we can just extract it from the histogram data that is discussed in the main text: We select from the already-known forward-CT excitations the one with lowest energy.

From Figure S11 we can draw several conclusions which support our line of argument in the main text. The main $\mathrm{Q}_{\mathrm{y}}$ energy level fluctuates between 1.6 and $1.8 \mathrm{eV}$, the mean 


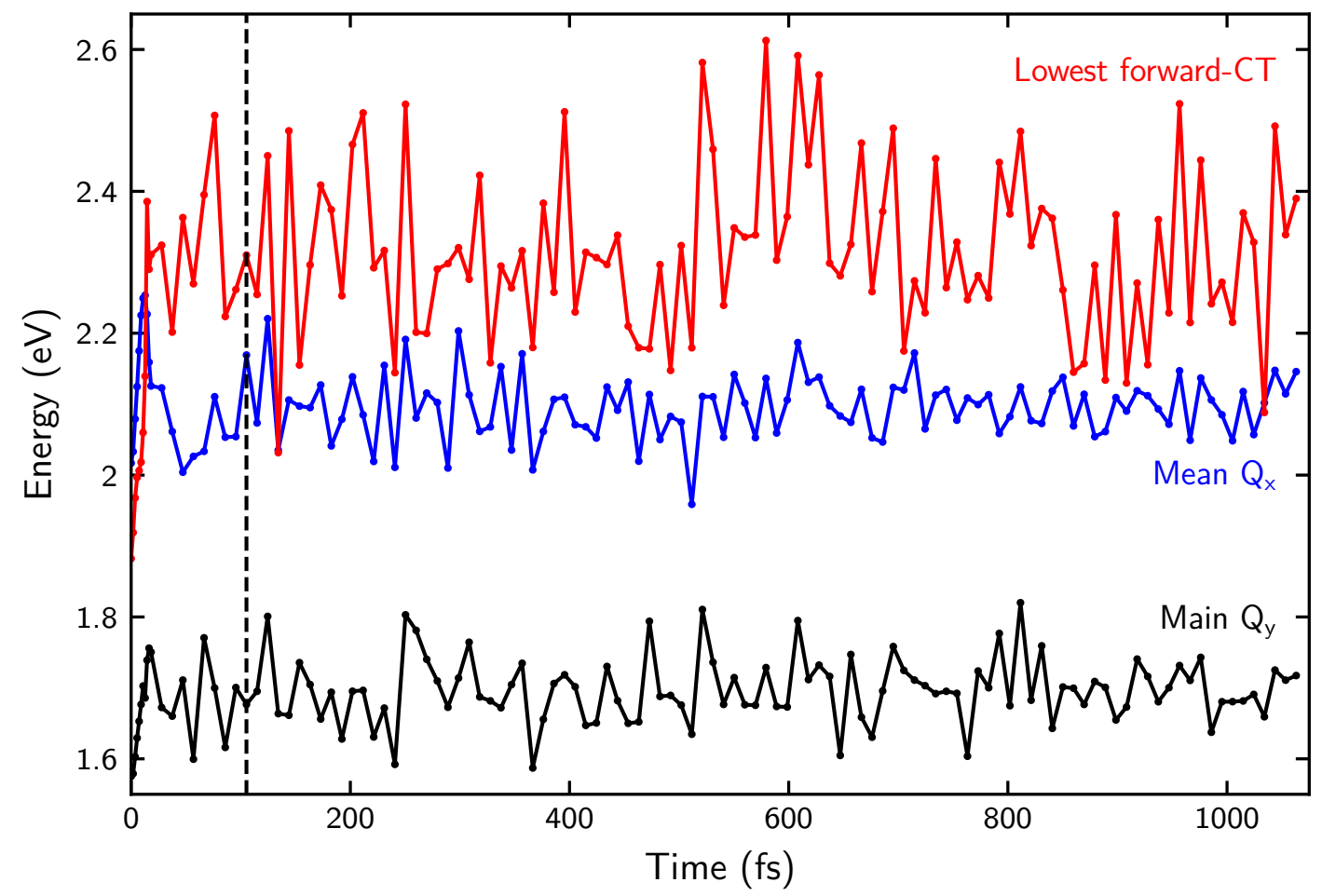

Figure S11: Time evolution of the lowest forward-CT excitation energy and energy levels representing the coupled $Q_{y}$ and $Q_{x}$ excitations (see text for their definitions). The dashed line (at $105.2 \mathrm{fs}$ ) marks the end of the equilibration phase and the beginning of the sampling for the histogram Figure 7 (main text).

$\mathrm{Q}_{\mathrm{x}}$ between 2.0 and $2.2 \mathrm{eV}$. The lowest forward-CT excitation is somewhat higher in energy; it lies mostly between 2.1 and $2.5 \mathrm{eV}$. Notably, there are a few snapshots where the lowest forward-CT energy is close to or even below the mean $Q_{x}$. By contrast, the main $\mathrm{Q}_{\mathrm{y}}$ and lowest forward-CT energies are separated by $\gtrsim 0.4 \mathrm{eV}$ during the whole BOMD run.

In the previous discussion we have excluded the data from the first $\sim 100 \mathrm{fs}$ (until the dashed line in Figure S11). During this time span, which we refer to as the "initial equilibration phase" in the main text, the pigment structures - initially in the X-ray geometry - equilibrate to the canonical ensemble mimicked by the MD simulation. The first few points in Figure S11 show this process. In the beginning, the energy levels are still close to the ones of the crystal structure, and, hence, do not represent the influence of the thermally driven nuclear motion. 


\section{Experimental Spectra}

A direct comparison of our calculated spectra to the available experimental absorption spectrum of the HbRC from [6] is difficult. Our study focuses on the electronic effects in the vicinity of the special pair and our simulations neither includes the complete protein environment nor the solvent. The latter can be expected to induce a global shift of the excitation energies. Moreover, our SPP-Acc system is limited to 4 out of $58 \mathrm{Bcl}$ pigments in total. Especially the $\mathrm{OH}-\mathrm{Chl} a$ pigments, whose spectral properties differ significantly from those of $\operatorname{Bcl} g[7,8]$ and which give rise to another absorption band peak at $\sim 1.85 \mathrm{eV}$ in the experimental spectrum, are absent in our current simulations. However, our calculations obtain qualitative agreement with the experimental peak positions of the $\mathrm{Q}_{\mathrm{y}}$ and $\mathrm{Q}_{\mathrm{x}}$ bands, $\sim 1.6 \mathrm{eV}$ and $\sim 2.2 \mathrm{eV}$, respectively. This is in line with earlier findings showing that TDDFT with non-local exchange-correlation approximations can describe the excitations of different Bcls with reasonable accuracy [9-11].

\section{Tables of the Excitation Spectra}

For the sake of completeness and transparency the following Tables S1 to S5 list the excitation energies and oscillator strengths of the relevant excitations of all spectra depicted in the main text and the Supporting Information of this article. We chose to present the excitations in the order in which they appear in the SPP-Acc system, i. e., first the (coupled) $\mathrm{Q}_{\mathrm{y}}$ excitations, then the forward-CT pair with lower energy, then the (coupled) $\mathrm{Q}_{\mathrm{x}}$ excitations, and finally the second forward-CT pair (for subsystems: as far as the excitations occur).

\section{References}

[1] I. Schelter, J. M. Foerster, A. T. Gardiner, A. W. Roszak, R. J. Cogdell, G. M. Ullmann, T. B. DE QueIroz, and S. Kümmel, Assessing density functional theory in real-time and real-space as a tool for studying bacteriochlorophylls and the light-harvesting complex 2, J. Chem. Phys. 151, 134114 (2019).

[2] M. Kasha, H. R. Rawls, and M. A. El-Bayoumi, The exciton model in molecular spectroscopy, Pure Appl. Chem. 11, 371 (1965).

[3] A. KöHler and H. BÄssler, Electronic Processes in Organic Semiconductors (Wiley-VCH Verlag GmbH \& Co. KGaA, May 2015).

[4] T. Körzdörfer, J. S. Sears, C. Sutton, and J.-L. Brédas, Long-range corrected hybrid functionals for $\pi$-conjugated systems: dependence of the rangeseparation parameter on conjugation length, J. Chem. Phys. 135, 204107 (2011).

[5] T. B. DE QueIroz and S. KÜMmeL, Charge-transfer excitations in low-gap systems under the influence of solvation and conformational disorder: exploring rangeseparation tuning, J. Chem. Phys. 141, 084303 (2014). 


\begin{tabular}{|c|c|c|c|c|c|}
\hline & SPP-Acc & SPP & Acc & $\mathrm{Bcl} g^{\prime} \&$ His & $\mathrm{Bcl} g \& \mathrm{Gln}$ \\
\hline$Q_{y}$ & $\begin{array}{l}1.389(0.423) \\
1.433(0.090) \\
1.510(0.708) \\
1.535(0.079) \\
1.707(0.016) \\
1.776(0.068)\end{array}$ & $\begin{array}{l}1.398(0.350) \\
1.446(0.023) \\
1.709(0.016) \\
1.774(0.077)\end{array}$ & $\begin{array}{l}1.534(0.613) \\
1.554(0.066)\end{array}$ & $1.567(0.265)$ & $1.543(0.322)$ \\
\hline CT & $\begin{array}{l}1.818(0.000) \\
1.847(0.001)\end{array}$ & & & & \\
\hline$Q_{x}$ & $\begin{array}{l}1.980(0.006) \\
1.985(0.026) \\
1.991(0.059) \\
2.002(0.061)\end{array}$ & $\begin{array}{l}1.992(0.016) \\
1.995(0.023)\end{array}$ & $\begin{array}{l}2.018(0.049) \\
2.020(0.066)\end{array}$ & $2.001(0.032)$ & $2.019(0.059)$ \\
\hline CT & $\begin{array}{l}2.187(0.000) \\
2.216(0.000)\end{array}$ & & & & \\
\hline
\end{tabular}

Table S1: Excitation energies (in $\mathrm{eV}$ ) and oscillator strengths (in parentheses) corresponding to Figure 4 (main text) and Figure S1. The excitations of the SPP and Accs, respectively, are arranged to indicate the correspondence to the excitations of the SPP-Acc system.

\begin{tabular}{c|ccc}
\hline & Original structure & Symmetrized I & Symmetrized II \\
\hline \multirow{6}{*}{$Q_{y}$} & $1.454(0.449)$ & $1.446(0.433)$ & $1.460(0.460)$ \\
& $1.499(0.090)$ & $1.496(0.085)$ & $1.502(0.094)$ \\
& $1.575(0.729)$ & $1.579(0.735)$ & $1.572(0.726)$ \\
& $1.600(0.080)$ & $1.603(0.078)$ & $1.598(0.076)$ \\
& $1.766(0.016)$ & $1.770(0.017)$ & $1.765(0.016)$ \\
& $1.831(0.061)$ & $1.834(0.063)$ & $1.830(0.060)$ \\
\hline \multirow{2}{*}{$\mathrm{CT}$} & $1.882(0.000)$ & $1.846(0.001)$ & $1.925(0.000)$ \\
& $1.894(0.001)$ & $1.847(0.001)$ & $1.927(0.001)$ \\
\hline \multirow{3}{*}{$\mathrm{Q}_{\mathrm{x}}$} & $2.012(0.015)$ & $2.010(0.009)$ & $2.013(0.049)$ \\
& $2.016(0.034)$ & $2.012(0.049)$ & $2.013(0.059)$ \\
& $2.017(0.082)$ & $2.035(0.050)$ & $2.018(0.062)$ \\
& $2.033(0.078)$ & $2.035(0.107)$ & $2.018(0.034)$ \\
\hline \multirow{2}{*}{$\mathrm{CT}$} & $2.240(0.000)$ & $2.214(0.000)$ & $2.283(0.000)$ \\
& $2.260(0.000)$ & $2.215(0.000)$ & $2.284(0.000)$ \\
\hline
\end{tabular}

Table S2: Excitation energies (in eV) and oscillator strengths (in parentheses) corresponding to Figure S5. 


\begin{tabular}{c|ccc|c}
\hline & \multicolumn{3}{|c|}{$\omega=0.171 \mathrm{a}_{0}^{-1}$} & $\omega=0.160 \mathrm{a}_{0}^{-1}$ \\
& $6-31 \mathrm{G}$ & $6-31 \mathrm{G}(\mathrm{d}, \mathrm{p})$ & $6-311 \mathrm{G}(\mathrm{d}, \mathrm{p})$ & $6-31 \mathrm{G}(\mathrm{d}, \mathrm{p})$ \\
\hline \multirow{4}{*}{$\mathrm{Q}_{\mathrm{y}}$} & $1.454(0.449)$ & $1.389(0.423)$ & $1.369(0.431)$ & $1.384(0.381)$ \\
& $1.499(0.090)$ & $1.433(0.090)$ & $1.410(0.094)$ & $1.435(0.077)$ \\
& $1.575(0.729)$ & $1.510(0.708)$ & $1.483(0.706)$ & $1.521(0.727)$ \\
& $1.600(0.080)$ & $1.535(0.079)$ & $1.509(0.076)$ & $1.544(0.090)$ \\
& $1.766(0.016)$ & $1.707(0.016)$ & $1.692(0.015)$ & $1.677(0.016)$ \\
& $1.831(0.061)$ & $1.776(0.068)$ & $1.758(0.063)$ & $1.751(0.076)$ \\
\hline \multirow{2}{*}{$\mathrm{CT}$} & $1.882(0.000)$ & $1.818(0.000)$ & $1.796(0.001)$ & $1.757(0.008)$ \\
& $1.894(0.001)$ & $1.847(0.001)$ & $1.823(0.001)$ & $1.786(0.001)$ \\
\hline \multirow{3}{*}{$\mathrm{Q}$} & $2.012(0.015)$ & $1.980(0.006)$ & $1.959(0.003)$ & $1.969(0.006)$ \\
& $2.016(0.034)$ & $1.985(0.026)$ & $1.965(0.025)$ & $1.974(0.026)$ \\
& $2.017(0.082)$ & $1.991(0.059)$ & $1.971(0.055)$ & $1.981(0.058)$ \\
& $2.033(0.078)$ & $2.002(0.061)$ & $1.982(0.057)$ & $1.993(0.060)$ \\
\hline \multirow{2}{*}{$\mathrm{CT}$} & $2.240(0.000)$ & $2.187(0.000)$ & $2.166(0.000)$ & $2.116(0.000)$ \\
& $2.260(0.000)$ & $2.216(0.000)$ & $2.194(0.000)$ & $2.146(0.000)$ \\
\hline
\end{tabular}

Table S3: Excitation energies (in eV) and oscillator strengths (in parentheses) corresponding to Figure S7 and Figure S9.

\begin{tabular}{c|cccc}
\hline & $\mathrm{His} \& \mathrm{H}_{2} \mathrm{O}-\mathrm{Gln}$ & $\mathrm{His}$ & $\mathrm{H}_{2} \mathrm{O}-\mathrm{Gln}$ & Vacuum \\
\hline \multirow{6}{*}{$\mathrm{Q}_{\mathrm{y}}$} & $1.454(0.449)$ & $1.455(0.447)$ & $1.452(0.454)$ & $1.454(0.452)$ \\
& $1.499(0.090)$ & $1.497(0.088)$ & $1.513(0.102)$ & $1.512(0.101)$ \\
& $1.575(0.729)$ & $1.575(0.713)$ & $1.586(0.707)$ & $1.584(0.699)$ \\
& $1.600(0.080)$ & $1.599(0.079)$ & $1.611(0.071)$ & $1.609(0.070)$ \\
& $1.766(0.016)$ & $1.768(0.017)$ & $1.752(0.016)$ & $1.754(0.017)$ \\
& $1.831(0.061)$ & $1.831(0.062)$ & $1.832(0.074)$ & $1.831(0.075)$ \\
\hline \multirow{2}{*}{$\mathrm{CT}$} & $1.882(0.000)$ & $1.724(0.001)$ & $2.386(0.001)$ & $2.223(0.001)$ \\
& $1.894(0.001)$ & $1.734(0.001)$ & $2.399(0.001)$ & $2.233(0.001)$ \\
\hline \multirow{3}{*}{$\mathrm{Q}$} & $2.012(0.015)$ & $2.014(0.024)$ & $2.023(0.071)$ & $2.076(0.022)$ \\
& $2.016(0.034)$ & $2.017(0.032)$ & $2.040(0.070)$ & $2.079(0.046)$ \\
& $2.017(0.082)$ & $2.073(0.066)$ & $2.076(0.009)$ & $2.081(0.033)$ \\
& $2.033(0.078)$ & $2.088(0.053)$ & $2.079(0.033)$ & $2.091(0.062)$ \\
\hline \multirow{2}{*}{$\mathrm{CT}$} & $2.240(0.000)$ & $2.078(0.015)$ & $2.732(0.001)$ & $2.570(0.000)$ \\
& $2.260(0.000)$ & $2.097(0.001)$ & $2.755(0.003)$ & $2.587(0.000)$ \\
\hline
\end{tabular}

Table S4: Excitation energies (in eV) and oscillator strengths (in parentheses) corresponding to Figure S8. 


\begin{tabular}{c|ccccc}
\hline & No env. & Phythyl & Trp & Trp-Thr-Tyr & Full env. \\
\hline \multirow{6}{*}{$\mathrm{Q}_{\mathrm{y}}$} & $1.454(0.449)$ & $1.457(0.468)$ & $1.453(0.431)$ & $1.451(0.440)$ & $1.408(0.418)$ \\
& $1.499(0.090)$ & $1.496(0.091)$ & $1.496(0.095)$ & $1.495(0.105)$ & $1.467(0.083)$ \\
& $1.575(0.729)$ & $1.561(0.661)$ & $1.572(0.780)$ & $1.555(0.788)$ & $1.550(0.765)$ \\
& $1.600(0.080)$ & $1.586(0.071)$ & $1.599(0.076)$ & $1.582(0.063)$ & $1.573(0.056)$ \\
& $1.766(0.016)$ & $1.774(0.019)$ & $1.716(0.001)$ & $1.764(0.022)$ & $1.727(0.020)$ \\
& $1.831(0.061)$ & $1.832(0.064)$ & $1.788(0.001)$ & $1.828(0.048)$ & $1.795(0.042)$ \\
\hline \multirow{2}{*}{$\mathrm{CT}$} & $1.882(0.000)$ & $1.838(0.002)$ & $1.763(0.020)$ & $1.837(0.001)$ & $1.837(0.001)$ \\
& $1.894(0.001)$ & $1.902(0.001)$ & $1.825(0.054)$ & $1.933(0.001)$ & $1.932(0.001)$ \\
\hline \multirow{4}{*}{$\mathrm{Q}$} & $2.012(0.015)$ & $2.013(0.004)$ & $2.006(0.012)$ & $2.002(0.012)$ & $1.978(0.016)$ \\
& $2.016(0.034)$ & $2.015(0.054)$ & $2.010(0.041)$ & $2.008(0.072)$ & $1.982(0.051)$ \\
& $2.017(0.082)$ & $2.022(0.050)$ & $2.016(0.076)$ & $2.010(0.050)$ & $2.006(0.080)$ \\
& $2.033(0.078)$ & $2.023(0.106)$ & $2.031(0.073)$ & $2.028(0.074)$ & $2.027(0.076)$ \\
\hline \multirow{2}{*}{ CT } & $2.240(0.000)$ & $2.203(0.000)$ & $2.066(0.002)$ & $2.204(0.000)$ & $2.193(0.000)$ \\
& $2.260(0.000)$ & $2.260(0.000)$ & $2.148(0.000)$ & $2.287(0.000)$ & $2.275(0.000)$ \\
\hline
\end{tabular}

Table S5: Excitation energies (in $\mathrm{eV}$ ) and oscillator strengths (in parentheses) corresponding to Figure 6 (main text) and Figure S10.

[6] A. Chauvet, J. Sarrou, S. Lin, S. P. Romberger, J. H. Golbeck, S. Savikhin, and K. E. REDDING, Temporal and spectral characterization of the photosynthetic reaction center from Heliobacterium modesticaldum, Photosynth. Res. 116, 1 (2013).

[7] M. Kobayashi, E. J. van de Meent, C. Erkelens, J. Amesz, I. Ikegami, and T. WatanaBe, Bacteriochlorophyll $g$ epimer as a possible reaction center component of heliobacteria, Biochim. Biophys. Acta 1057, 89 (1991).

[8] E. J. van de Meent, M. Kobayashi, C. Erkelens, P. A. van Veelen, J. AMESZ, and T. WATANABE, Identification of $8^{1}$-hydroxychlorophyll $a$ as a functional reaction center pigment in heliobacteria, Biochim. Biophys. Acta 1058, 356 (1991).

[9] D. Sundholm, A density-functional-theory study of bacteriochlorophyll b, Phys. Chem. Chem. Phys. 5, 4265 (2003).

[10] J. Linnanto and J. Korppi-Tommola, Quantum chemical simulation of excited states of chlorophylls, bacteriochlorophylls and their complexes, Phys. Chem. Chem. Phys. 8, 663 (2006).

[11] Z.-L. Cai, M. J. Crossley, J. R. Reimers, R. Kobayashi, and R. D. Amos, Density Functional Theory for Charge Transfer: The Nature of the N-Bands of Porphyrins and Chlorophylls Revealed through CAM-B3LYP, CASPT2, and SACCI Calculations, J. Phys. Chem. B 110, 15624 (2006). 